\title{
PROYECTO EDUMOBSPITALARIOS: DESARROLLO PROFESIONAL DOCENTE E INNOVACIÓN CON M- LEARNING EN AULAS HOSPITALARIAS
}

\author{
EDUMOBSPITALARIOS PROJECT: TEACHER PROFESSIONAL \\ DEVELOPMENT AND INNOVATION WITH M-LEARNING IN \\ HOSPITAL CLASSROOMS
}

\author{
José Luis Serrano Sánchez; jl.serranosanchez@um.es \\ Linda Castañeda Quintero; lindacq@um.es \\ Universidad de Murcia
}

\section{RESUMEN}

En este artículo se presentan los resultados preliminares del Proyecto EDUMOBSPITALARIOS, una iniciativa de investigación-acción desarrollada por el Grupo de Investigación de Tecnología Educativa de la Universidad de Murcia (GITE) y el Equipo de Atención Educativa Hospitalaria y Domiciliaria (EAEHD) de la Región de Murcia. Este estudio tuvo como principal finalidad mejorar las dinámicas habituales de innovación en la atención educativa hospitalaria, mediante la implementación de un proceso de desarrollo profesional de docentes de aulas hospitalarias.

Concretamente, se diseñó, implementó y evaluó un plan de formación basado en seminarios de descubrimiento tecnológico y creación didáctica conjunta. Además se creó un metacatálogo de Apps del entorno Android para uso educativo y un catálogo de actividades didácticas enriquecidas con tecnologías móviles.

PALABRAS CLAVE: Desarrollo profesional docente, aulas hospitalarias, proyecto, formación, aprendizaje móvil.

\section{ABSTRACT}

In this paper we present the main results of EDUMOBSPITALARIOS Project, an action research initiative developed by the Research Group of Educational Technology at the University of Murcia (GITE) in conjunction with the Education and Care Hospital Equipment and Homecare (EAEHD) of the Region of Murcia. The purpose of the study 
has been to improve the usual innovation dynamic in hospital schools hospitals through the implementation of a process of teacher's professional development. In particular, it is designed, implemented and evaluated a training plan based on seminars of technological exploration and educational co-creation, during which the participants developed, collaboratively and sequentially, a meta-catalogue of Apps for educational use (Android) and a catalogue of mobile-technology enhanced educational activities, for hospital schools.

KEYWORDS: Teacher professional development, hospital schools, project, training, mobile learning.

\section{INTRODUCCIÓN}

La implementación de Tecnologías de la Información y la Comunicación (TIC) en los procesos de aprendizaje y motivación del alumnado de aulas hospitalarias (en adelante $\mathrm{AAHH}$ ), es una de las estrategias que se ha revelado como más interesante a la hora de mejorar la atención educativa integral, quedando integrada en el uso de metodologías flexibles, diversas y sobre todo adaptadas a las necesidades (edad, intereses, nivel educativo y tipo de lesión o enfermedad) del alumnado hospitalizado (Campbell, y St Leger, 2006; Cook, 2005; Jones y McDougall, 2010; Nisselle, Hanns, Green y Jones 2012; Serrano y Prendes, 2014, entre otros).

La dificultad de este tipo de implementación (estudiada en profundidad en Serrano, 2013), radica no solo en las ya complejas relaciones inherentes a cualquier proceso de enseñanza-aprendizaje (Williams, Mackness, y Gumtau, 2012; Adell y Castañeda, 2013), sino que en el contexto hospitalario la finalidad última es mejorar la calidad de vida del menor y utilizar el aprendizaje para sanar durante el periodo de convalecencia.

En este marco, entendemos que la integración curricular de TIC en AAHH obedece de forma complementaria a cuatro dimensiones principales que consideramos que deben ser abordadas de forma sistemática y paralela en cualquier iniciativa que se proponga en este contexto educativo: contexto, formación y asesoramiento, principios metodológicos y herramientas telemáticas (Serrano y Prendes, 2015).

En consecuencia, desde el Grupo de Investigación de Tecnología Educativa de la Universidad de Murcia (en adelante GITE) hemos venido desarrollando en estrecha colaboración con el Equipo de Atención Educativa Hospitalaria y Domiciliaria de la Región de Murcia (en adelante EAEHD), una línea de investigación-acción centrada en la formación docente para el diseño y la implementación de buenas prácticas con TIC en la educación hospitalaria (en Prendes, Castañeda y Serrano, 2014) se describieron las principales iniciativas de los últimos años), en la que se ha intentado abordar cada una de esas dimensiones de forma explícita, donde los ejes fundamentales han sido "el uso de las TIC en el ámbito académico-formativo, la focalización en el diseño y evaluación de metodologías y una atención especial a los procesos de desarrollo profesional docente" (p. 94). 
A continuación presentamos el procedimiento seguido y resultados de una de las iniciativas que configuran esa línea de investigación-acción, en la que la dimensión formación y asesoramiento adquiere una especial relevancia, puesto que entiende que (una vez provistos unos mínimos de equipamiento y contextualización de la acción con TIC) la promoción de un ambiente de desarrollo profesional docente continuo, es la clave para conseguir cambios duraderos en las prácticas educativas de una institución (Fullan, 2002). Sin embargo, esta dimensión formativa y de asesoramiento intenta huir de los planteamientos más tradicionales, y por lo que se propone como un "proceso de adquisición basado en la participación y en la reflexión práctica" (Castañeda y Adell, 2011, p.83) en la que los docentes traten de resolver de manera colaborativa sus necesidades a partir de la investigación y reflexión sobre su propia práctica con otros colegas, tanto de dentro del aula como de fuera y se consiga la creación de espacios en los que compartir prácticas, experiencias y promover la colaboración para nuevas iniciativas. Esta creación de redes profesionales, dedicadas a la innovación con TIC, debería contribuir por un lado al desarrollo profesional de sus participantes, y por otro a facilitar el acceso a la información para la comprensión de la atención educativa en el hospital (Castañeda y Sánchez, 2012; González et al., 2011).

\section{PROYECTO EDUMOBSPITALARIOS}

EI Proyecto EDUMOBSPITALARIOS: TIC para la creación de espacios educativos sociales en las aulas hospitalarias (http://www.um.es/aulashospitalarias/mo/), es un estudio conjunto de investigación e Innovación educativa que tiene como finalidad la creación de espacios educativos y sociales con el apoyo y el uso de las tecnologías móviles en las AAHH de la Región de Murcia. Ha sido cofinanciado por la Comunidad Autónoma de la Región de Murcia y la Universidad de Murcia, y dirigido por Linda Castañeda.

La investigación consistió en la implementación de un proceso de desarrollo profesional docente en los profesionales de las AAHH de la Región de Murcia (basado en un modelo concreto), que supusiera un cambio duradero en las dinámicas de innovación habituales, usando como reto la implementación didáctica de tecnologías móviles (m-learning) en el contexto de la atención educativa para niños enfermos.

Se llevaron a cabo tres iniciativas principales que se convirtieron también en nuestros objetivos de investigación. Iniciativas que se implementaron de forma secuencial:

1. Organizar un catálogo de aplicaciones móviles que pudieran servir de apoyo y enriquecimiento al proceso educativo del alumnado hospitalizado.

2. Diseñar e implementar un plan de desarrollo profesional docente en torno al uso de aplicaciones móviles en el contexto de la educación hospitalaria.

3. Construir un catálogo de propuestas didácticas que implementen de forma sustancial Apps móviles en la educación hospitalaria.

La finalidad de este artículo es presentar las tareas realizadas para el logro de los objetivos descritos anteriormente además de mostrar los principales resultados 
teniendo en cuenta la opinión de los participantes.

\section{MÉTODO}

El procedimiento de investigación seguido estuvo supeditado por las características de la investigación evaluativa, la cual queda enmarcada bajo el paradigma pragmático, haciendo un uso combinado de métodos cuantitativos y cualitativos, con el objetivo de obtener información relevante y útil que sirviera para la consecución de los objetivos, además de proporcionar recomendaciones de mejora en el contexto de estudio (Correa, Puerta y Restrepo, 2002; Escudero, 2011; García, 2012; Ramos, 2011). Los seis participantes (el total de maestros de AAHH de la Región de Murcia) fueron seleccionados por muestreo no probabilístico intencional con un criterio específico, que fuesen maestros de AAHH. Por las características de la población, y como es habitual en las investigaciones evaluativas, los resultados obtenidos no se pueden generalizar más allá del contexto de investigación. Sin embargo, el procedimiento seguido se puede considerar también como un resultado de estudio y que sí sería aplicable para la resolución de problemas semejantes.

\subsection{Instrumento de análisis de trabajo}

Ningún instrumento diseñado anteriormente se ajustaba a la finalidad de nuestra investigación, ya que no existe un precedente en el que se haya diseñado un plan de formación con características similares al nuestro. Por este motivo se decidió diseñar un cuestionario para evaluar la experiencia de cada uno de los seminarios.

A través de este instrumento se recogió la valoración y opinión de los maestros de las AAHH participantes en el proyecto en relación al diseño y desarrollo de los seminarios. Consta de un total de 22 ítems, de los cuales 18 son cerrados y 4 abiertos. El tabla 1 se recoge información sobre la relación entre los bloques, categorías, opciones de respuesta y número de ítem del cuestionario.

\begin{tabular}{|c|c|c|c|}
\hline Bloques & Categorías & Opciones de respuesta & $\begin{array}{l}\text { No de } \\
\text { ítem }\end{array}$ \\
\hline \multirow{3}{*}{$\begin{array}{c}\text { Datos de } \\
\text { identificación }\end{array}$} & Sexo & Hombre/Mujer & 1 \\
\hline & Edad & Abierto & 2 \\
\hline & $\begin{array}{c}\text { Experiencia } \\
\text { como docente }\end{array}$ & Abierto & $3,4,5$ \\
\hline \multirow{3}{*}{$\begin{array}{l}\text { Seminario de } \\
\text { formación } \\
\text { presencial }\end{array}$} & $\begin{array}{l}\text { Actuaciones } \\
\text { realizadas en } \\
\text { el seminario }\end{array}$ & $\begin{array}{c}\text { No se realizó/muy } \\
\text { adecuada/inadecuada/adecuada/muy } \\
\text { adecuada }\end{array}$ & 6 \\
\hline & $\begin{array}{l}\text { Características } \\
\text { del formador }\end{array}$ & Muy baja/baja/alta/muy alta & 7 \\
\hline & $\begin{array}{l}\text { Calidad del } \\
\text { seminario }\end{array}$ & Muy baja/baja/alta/muy alta & 8 \\
\hline
\end{tabular}




\begin{tabular}{|c|c|c|c|}
\hline \multirow{3}{*}{$\begin{array}{l}\text { Diseño de } \\
\text { actividades }\end{array}$} & Dificultad & Muy baja/baja/alta/muy alta & 9 \\
\hline & $\begin{array}{l}\text { Gestión } \\
\text { actividades } \\
\text { diseñadas }\end{array}$ & $\begin{array}{c}\text { Sí/No } \\
\text { Pertinente/no pertinente }\end{array}$ & $\begin{array}{l}10 \\
11\end{array}$ \\
\hline & $\begin{array}{l}\text { Tipo } \\
\text { actividades } \\
\text { diseñadas }\end{array}$ & Elección múltiple & 12 \\
\hline Implementación & $\begin{array}{l}\text { Efecto de las } \\
\text { TIC en el } \\
\text { aprendizaje }\end{array}$ & No lo sé/nada/poco/bastante/mucho & 13 \\
\hline \multirow{3}{*}{ Asesoramiento } & $\begin{array}{l}\text { Necesidad del } \\
\text { asesoramiento }\end{array}$ & $\begin{array}{c}\text { Muy } \\
\text { necesario/innecesario/necesario/muyn } \\
\text { ecesario }\end{array}$ & 14 \\
\hline & $\begin{array}{l}\text { Consultas } \\
\text { realizadas }\end{array}$ & $\begin{array}{c}\text { Ninguna, pocas, bastantes, muchas } \\
\text { Nunca, casi nunca, casi siempre, } \\
\text { siempre }\end{array}$ & $\begin{array}{l}15 \\
16,17\end{array}$ \\
\hline & $\begin{array}{c}\text { Tipo de } \\
\text { asesoramiento }\end{array}$ & Presencial/virtual/ambos & 18 \\
\hline $\begin{array}{l}\text { Valoración } \\
\text { general }\end{array}$ & Valoración & $\begin{array}{l}\text { Pésima/baja/alta/excelente } \\
\text { Elección múltiple } \\
\text { Abierto }\end{array}$ & $\begin{array}{l}20 \\
21 \\
22\end{array}$ \\
\hline
\end{tabular}

Tabla 1. Relación de bloques, categorías, opciones de respuesta y número de ítems del instrumento

El cuestionario se diseñó mediante la herramienta "Encuestas" de la Universidad de Murcia. Desde la siguiente URL se puede acceder abiertamente al instrumento en modo previsualización: https://goo.gl/nBUeln

Fue validado con la técnica de juicio de expertos. Proceso que consistió en solicitar a tres expertos en el área de la tecnología educativa la demanda de un juicio y opinión hacia el instrumento diseñado. Para ello se creó una ficha de validación en la que se detalló el objetivo del instrumento y las instrucciones precisas para proceder a su validación. Cada experto tuvo que valorar la pertinencia y la claridad de cada uno de los ítems usando la escala de 1 a 3 (siendo 1: bajo; 2: medio; 3: alto). Además tenían la posibilidad de hacer sugerencias y observaciones.

Una vez que se recibieron los informes de valoración del instrumento, el equipo de investigación triángulo la información y procedió a mejorar el cuestionario teniendo en cuenta el trabajo realizado por los expertos.

\subsection{Procedimiento}

En este apartado mostramos las principales tareas realizadas, su relación con los objetivos de investigación y los principales resultados: diseño de un metacatálogo de aplicaciones móviles para Android; diseño y evaluación de un plan de desarrollo profesional docente; creación de un catálogo de propuestas didácticas con el uso de 
Apps. Las iniciativas centrales de este proyecto se abordaron secuencialmente, y respondieron a una serie de tareas (Figura 1 ) que se presentan a continuación:

- Definición de la dinámica - Seminario Apps Escritura - Seminario Apps Dibujo - Seminario Apps Vídeo - Seminario Apps Imágen fija - Seminario Apps Mapas mentales

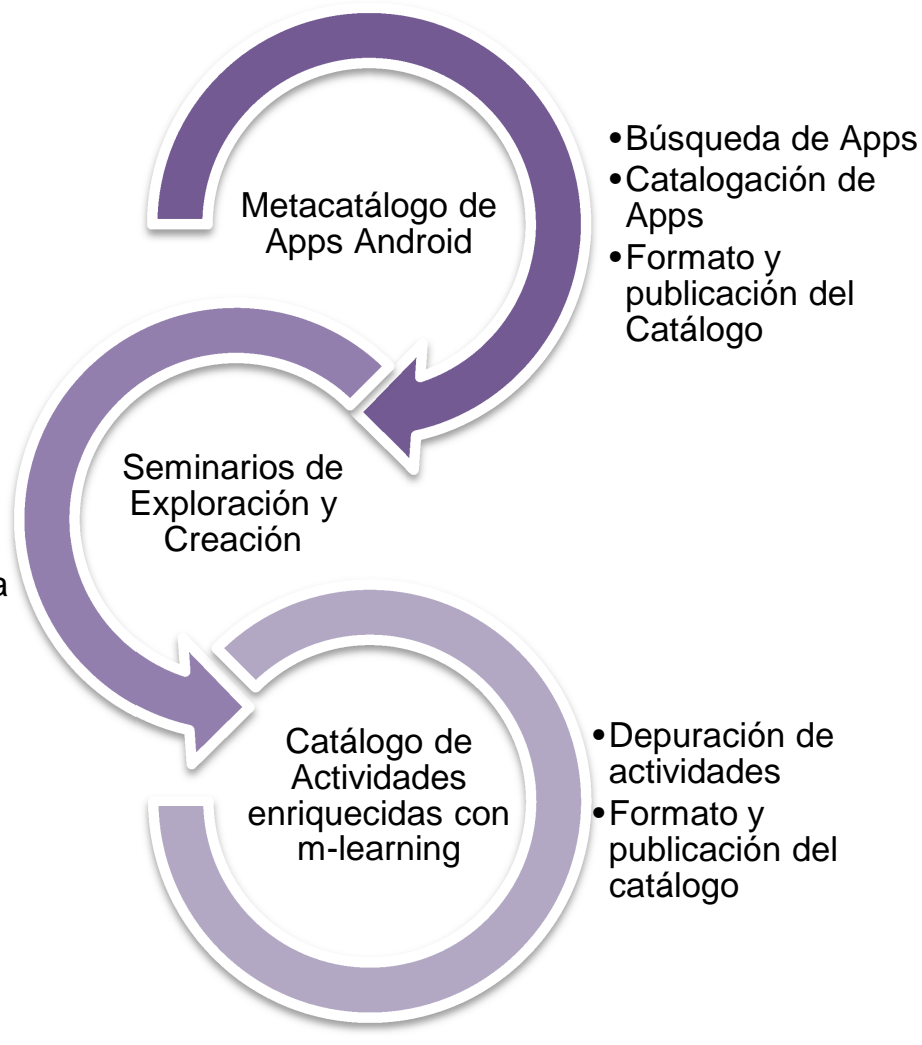

Figura 1. Objetivos y tareas, proyecto EDUMOBSPITALARIOS

\subsubsection{Organización del metacatálogo de aplicaciones móviles}

Para la consecución del primer objetivo del proyecto se realizó una estrategia de trabajo en la que se combinaron los esfuerzos de los dos grupos implicados (EAEHD-GITE) y se concretaron en 3 tareas:

\section{Tarea 1. Búsqueda de Apps para la educación}

Cada uno de los miembros del equipo realizó un listado de Apps. Se trataba de una lista en bruto, por lo que no necesariamente requería un proceso de selección sobre su pertinencia o no. En este listado sí era necesario indicar la proveniencia de la lista de Apps, la URL donde se incluye dicha lista, así como el nombre del compilador. Los datos se incluyeron en una hoja de cálculo compartida ${ }^{1}$.

\section{Tarea 2. Catalogación de las Apps}

Teniendo en cuenta los resultados de la tarea 1, procedimos a la clasificación y categorización de las Apps. En esta ocasión solo participamos los investigadores

\footnotetext{
${ }^{1}$ Listado de apps para la educación: $\underline{\text { https://goo.gl/Hs4yUb }}$
} 
del GITE. Una vez depurado el documento compartido, se repartieron las listas de Apps entre los investigadores. Para la clasificación de las aplicaciones se creó un nuevo documento ${ }^{2}$ compartido en Google Drive, en el que se indicó el nombre de la App, del listado/catálogo en el que se había encontrado, si requiere registro, tipo de código-formato de la App, si la App está vinculada a alguna asignatura del currículum y la edad recomendada de usuario.

En caso de que existieran listados con cientos de Apps, se entendió que no era posible y necesario reclasificarlos todos y se acordó seguir el siguiente criterio de selección: se escogería el top 10 de cada formato (texto, vídeo...) teniendo en cuenta aquellas que más se ajustaban a las características de la atención educativa hospitalaria (ver en Serrano y Prendes, 2014) y a la finalidad de la investigación. Los ránkines ofrecidos por los propios listados también fueron tenidos en cuenta para la selección de las Apps.

\section{Tarea 3. Organización y distribución del catálogo}

Para la organización y libre distribución del catálogo de Apps, se optó por un formato de publicación que pudiera ser simple, permitiendo un mantenimiento sencillo y que a la vez lo hiciera lo más usable posible a usuarios externos. Se utilizó la misma estrategia seguida ya en otros catálogos desarrollados por el GITE, es decir, la creación de una hoja de datos con las categorías depuradas y su publicación en la página web del proyecto con un plugin de publicación de tablas para WordPress (TablePress). El catálogo ${ }^{3}$ de Apps creado tras las dos tareas anteriores se encuentra accesible en la página Web del proyecto, y como se puede apreciar en la figura 2 se crearon también las páginas específicas donde se alojan cada uno de los catálogos de aplicaciones referidas a herramientas concretas: Apps para trabajar texto, dibujo, imagen fija, vídeo, videoconferencias, presentaciones visuales y mapas mentales.

\footnotetext{
${ }^{2}$ Listado de la catalogación de las apps para la educación: $\underline{\text { https://goo.gl/vWYVvi }}$

${ }^{3}$ Acceso al catálogo de apps: $\underline{\text { http://goo.gl/ZHmclg }}$
} 
Figura 2. Catálogos Apps Android del Proyecto EDUMOBSPITALARIOS

De la misma forma, esta iniciativa sirvió como germen de un catálogo específico de Apps Android para la enseñanza que aparece en el repositorio de "catálogos" gestionados por el GITE (Figura 3) y que continúa siendo actualizado y revisado periódicamente con una perspectiva más genérica (no solo especializada en el contexto educativo hospitalario).

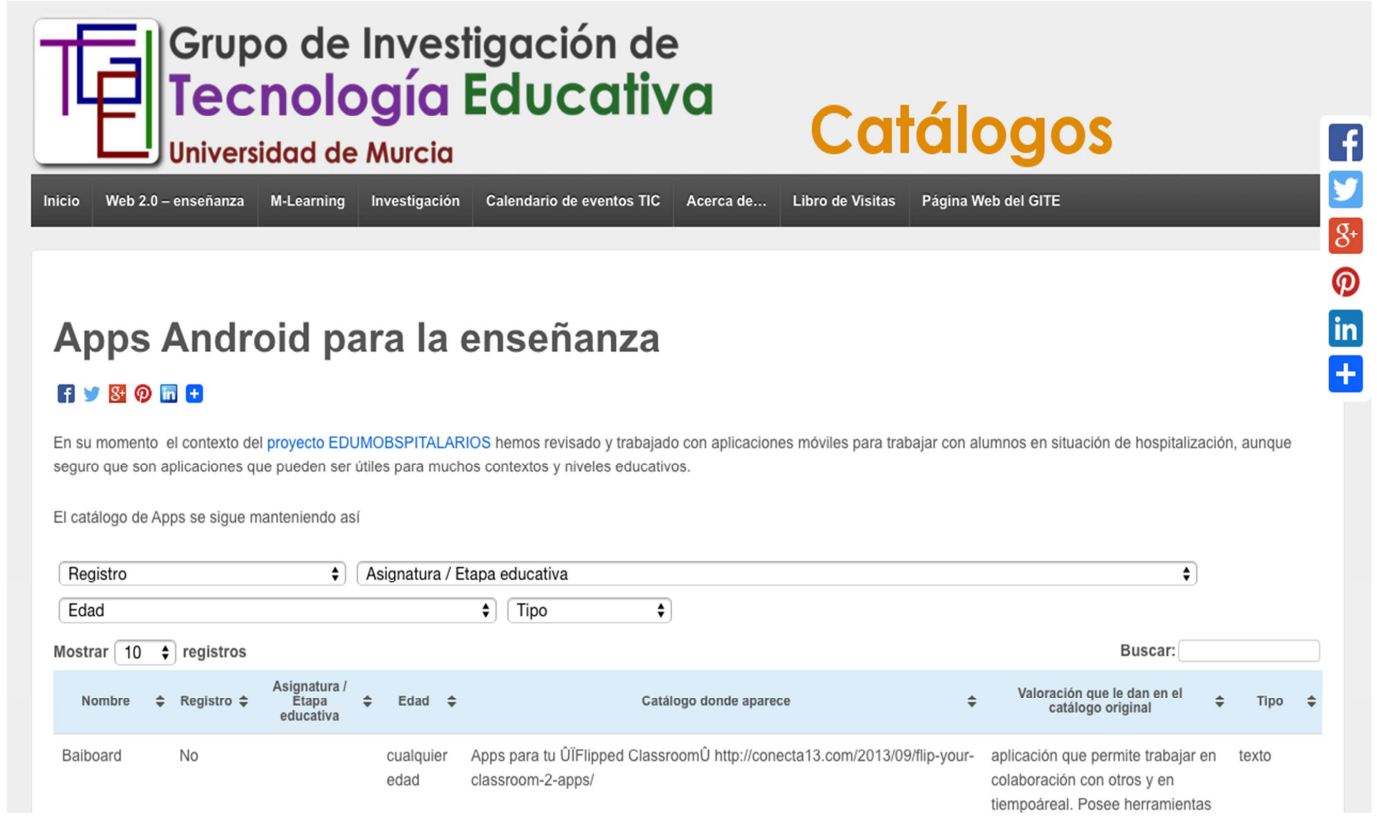

Figura 3. Catálogos GITE-Apps Android

\subsubsection{Diseño e implementación de un plan de desarrollo profesional docente}

El eje central del proyecto fue el diseño, implementación y evaluación de un plan de formación en formato seminarios de exploración, creación y participación conjunta.

Estas sesiones de formación se diseñaron desde la concepción de que los maestros participantes debían desarrollar su competencia digital teniendo en cuenta sus necesidades y las características de su realidad. Así, la dinámica seguida en estos seminarios de formación pretendía propiciar la elaboración de actividades con el uso de Apps para tablets, generando de esta manera espacios creativos para la resolución de necesidades de los maestros participantes en el estudio.

\section{Tarea 4. Propuesta, discusión y consenso de la dinámica a seguir}

Diseñado el catálogo de Apps, se inició la segunda parte de este proyecto con una primera reunión presencial del equipo de trabajo: maestros e investigadores. El seminario se llevó a cabo en el aula hospitalaria del hospital Virgen de la Arrixaca de Murcia. 
La principal finalidad de esta primera sesión de trabajo fue presentar y discutir con los maestros del EAEHD el plan de formación diseñado en formato seminarios. Cada uno de ellos (uno por mes) sería coordinado por un investigador del GITE, convirtiéndose además en el tutor de los docentes durante el mes siguiente para tratar de dinamizar el trabajo del grupo (que pretende convertirse en un grupo de colaboración) y de resolver cuantas dudas pudieran surgir. Los profesores de las $\mathrm{AAHH}$, por su parte, tenían la responsabilidad de asumir un papel activo dentro de los seminarios, además de trabajar intensamente en procesos de aprendizaje por descubrimiento, coevaluación de actividades, implementación de secuencias en escenarios reales y propuesta de continuidad. Además, en esta sesión se decidieron cuáles eran los formatos que más interesaban a los docentes y el orden en que preferían abordarlos teniendo en cuenta las necesidades y la propia programación de las AAHH.

Así, se decidió que la secuencia básica a seguir en cada seminario fuese la siguiente: el facilitador del seminario seleccionaría 4-5 Apps de uno de los formatos (texto, vídeo, dibujo...) teniendo en cuenta el catálogo diseñado en tareas anteriores y buscaría ejemplos de uso educativo de dichas Apps para mostrarlos a los profesores a modo de introducción al inicio de cada seminario. Posteriormente, los maestros, con ayuda del investigador, diseñarían actividades con las Apps para su contexto de trabajo e implementarían alguna(s) de esas actividades, con el alumnado hospitalizado.

Además de clarificar esta dinámica de trabajo, durante esta primera reunión se resolvieron otras cuestiones que precisaban del consenso de todo el equipo: el proceso de evaluación, el formulario ${ }^{4}$ de registro de actividades diseñadas durante los seminarios y una selección de los formatos de Apps del catálogo para trabajar en los seminarios.

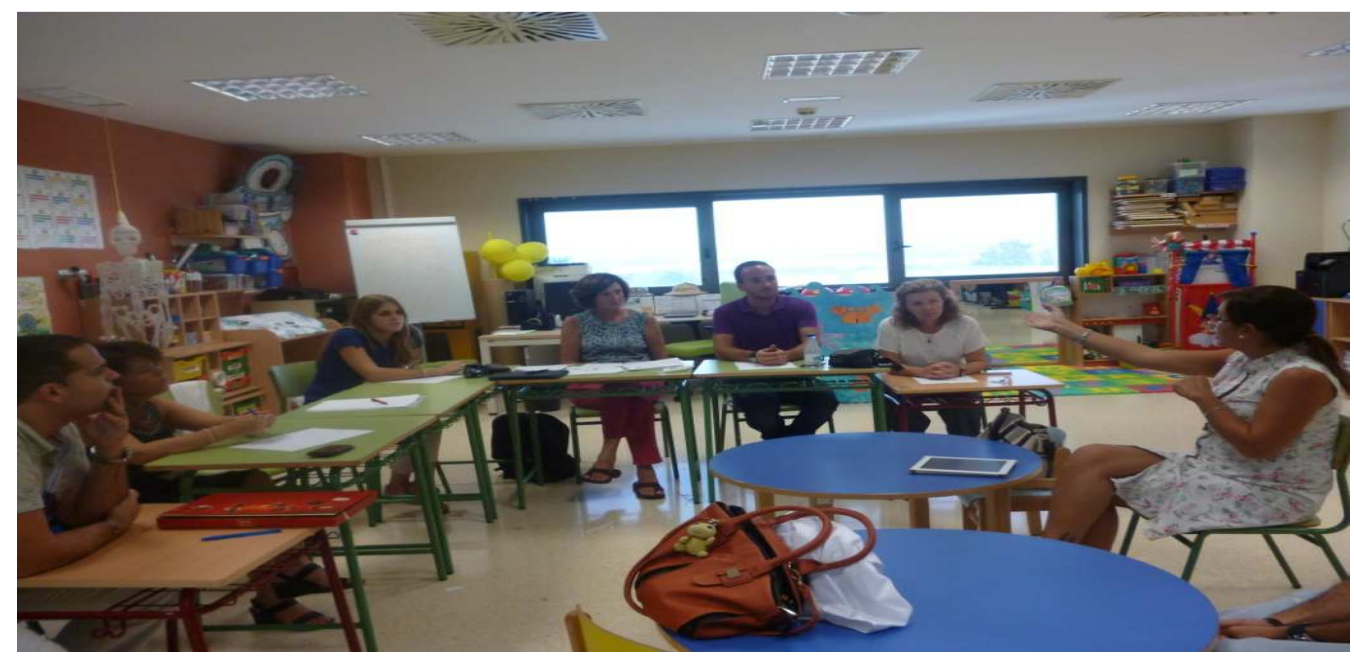

Figura 3. Durante la realización de la tarea 4

\footnotetext{
${ }^{4}$ Acceso disponible en: https://goo.gl/86XAP6
} 
En las tareas 5 a 9, se llevaron a cabo los seminarios de trabajo sobre el uso de Apps para trabajar la escritura, dibujo, vídeo, imagen y mapas mentales. A continuación, en la tabla 1 , se incluye la información y los recursos más relevantes de cada una de las sesiones de formación.

\begin{tabular}{|c|c|c|c|}
\hline Tarea & $\begin{array}{c}\text { Formato } \\
\text { App }\end{array}$ & Otros recursos & Apps y URLs \\
\hline & Escritura & $\begin{array}{c}\text { Claves para el uso del } \\
\text { vídeo: } \\
\text { https://goo.gl/knJ1Uk } \\
\text { Ejemplos de proyectos } \\
\text { y buenas prácticas: } \\
\text { https://goo.gl/pn4U2y }\end{array}$ & $\begin{array}{c}\text { Google Drive: } \\
\text { https://goo.gl/540GhZ } \\
\text { Blogger: } \\
\frac{\text { https://goo.gl/Kuzy4f }}{\text { Acrostic }} \\
\text { Poems:http://goo.gl/Wszqyp } \\
\frac{\text { Theme Poems: }}{\text { http://goo.gl/c98pXF }} \\
\text { Timeline: } \\
\text { http://goo.gl/NEaysd }\end{array}$ \\
\hline №6 & Dibujo & $\begin{array}{l}\text { Presentación visual: } \\
\text { http://goo.gl/VCoi5m }\end{array}$ & $\begin{array}{l}\text { Princesas para } \\
\text { colorear: } \\
\text { https://goo.gl/ThNCBS } \\
\text { SyncSpace: } \\
\frac{\text { https://goo.gl/PNJOST }}{\text { Quiver: }} \\
\frac{\text { https://goo.gl/Dw3hmy }}{\text { Skitch: } \frac{\text { https://goo.gl/TA90c }}{\text { Divertido Picture }}} \\
\text { Maker: } \underline{\text { https://goo.gl/xm3ecl }}\end{array}$ \\
\hline №7 & Vídeo & $\begin{array}{c}\text { Teachem: } \\
\frac{\text { http://goo.gl/6ylNLS }}{\text { Namathis: }} \\
\frac{\text { http://goo.gl/3VF5Qk }}{\text { Screencast-O-Matic: }} \\
\frac{\text { http://goo.gl/pQ2dcY }}{\text { Ejemplos: }} \\
\frac{\text { https://goo.gl/WGcNIO }}{\text { Experiencias Flipped }} \\
\text { Classroom: } \\
\frac{\text { http://goo.gl/100pds }}{\text { Presentación visual: }} \\
\text { http://goo.gl/DWn6XY }\end{array}$ & $\begin{array}{c}\text { Lensso Create: } \\
\frac{\text { https://goo.gl/NN6qc }}{\text { Magisto: }} \\
\frac{\text { https://goo.gl/gE1WS }}{\text { VideoShowLab: }} \\
\frac{\text { https://goo.gl/SbcLiV }}{\text { Video }} \\
\text { Converter: } \\
\text { https://goo.gl/xGMM5 }\end{array}$ \\
\hline №8 & Imagen & $\begin{array}{l}\text { Presentación visual: } \\
\text { http://goo.gl/uWgj9o }\end{array}$ & $\begin{array}{c}\text { Molecule Viewer } \\
\text { 3D: } \frac{\text { https://goo.gl/RW3kCR }}{\text { Visual Anatomy: }}\end{array}$ \\
\hline
\end{tabular}




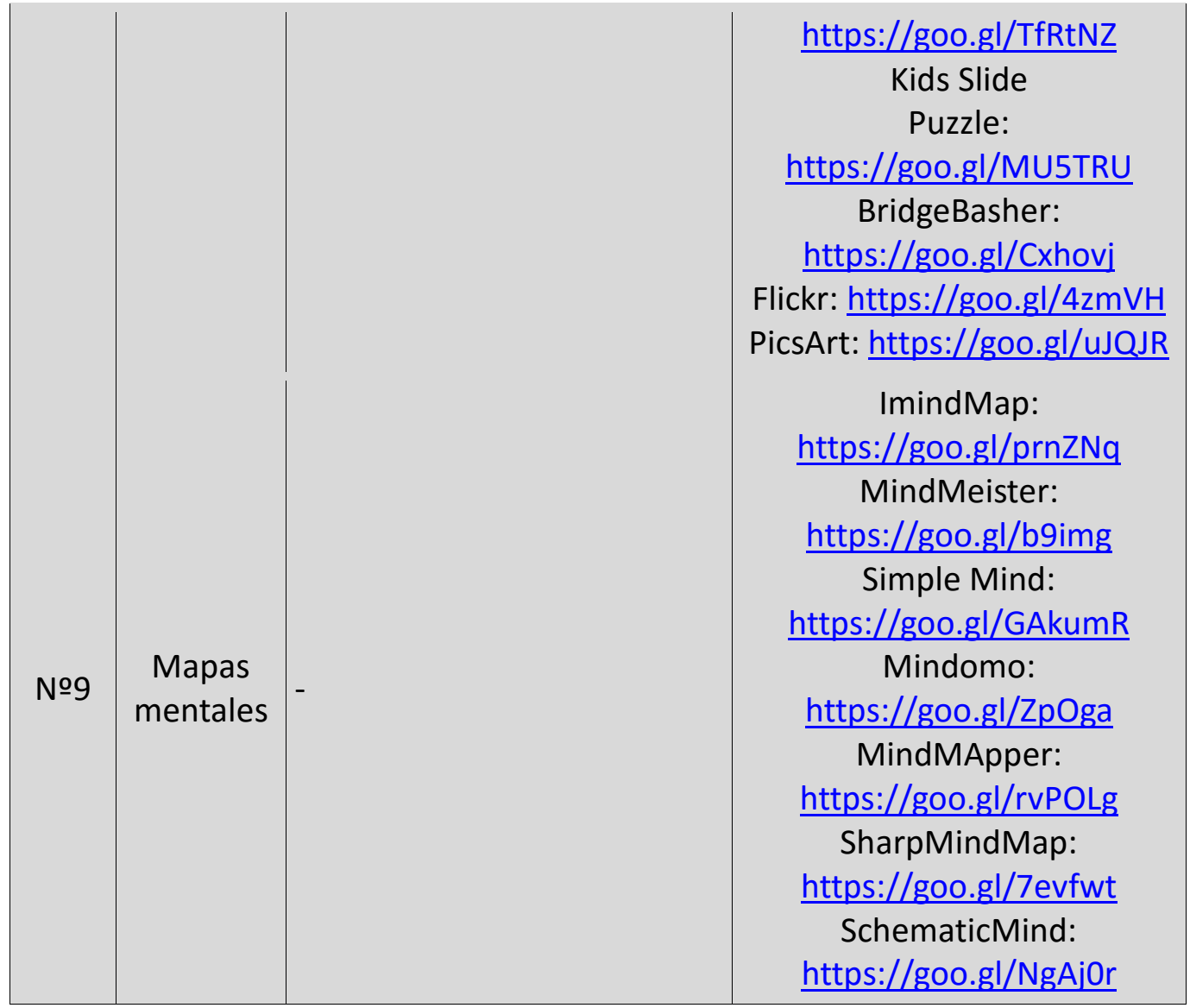

Tabla 2. Resumen y recursos de los seminarios continuos de formación

\subsubsection{Catálogo de propuestas didácticas con Apps móviles}

Una de las claves del proceso de formación y desarrollo profesional que planteábamos en este proyecto era hacer a los maestros partícipes y protagonistas de un cambio que debía ser didáctico más que tecnológico. En este sentido los seminarios no se plantearon como acciones puntuales, sino como seminarios de trabajo de larga duración en el tiempo y con una tarea clara a desarrollar por cada uno de ellos: proponer e implementar actividades didácticas enriquecidas por el entorno móvil en su actuación diaria en las AAHH

El valor innegable de estas actividades radicaba en su propia naturaleza, pero especialmente, en el hecho de que hubieran sido desarrolladas, probadas y catalogadas por los mismos docentes con el asesoramiento del responsable de cada seminario.

El catálogo de actividades se encuentra alojado en la Web del proyecto en la siguiente dirección: $\underline{\text { http://goo.gl/tdwDqN }}$

Con esta secuencia de actividades, el cronograma final del proyecto respondió básicamente a la línea de tiempo que proponemos en la figura 4 . 


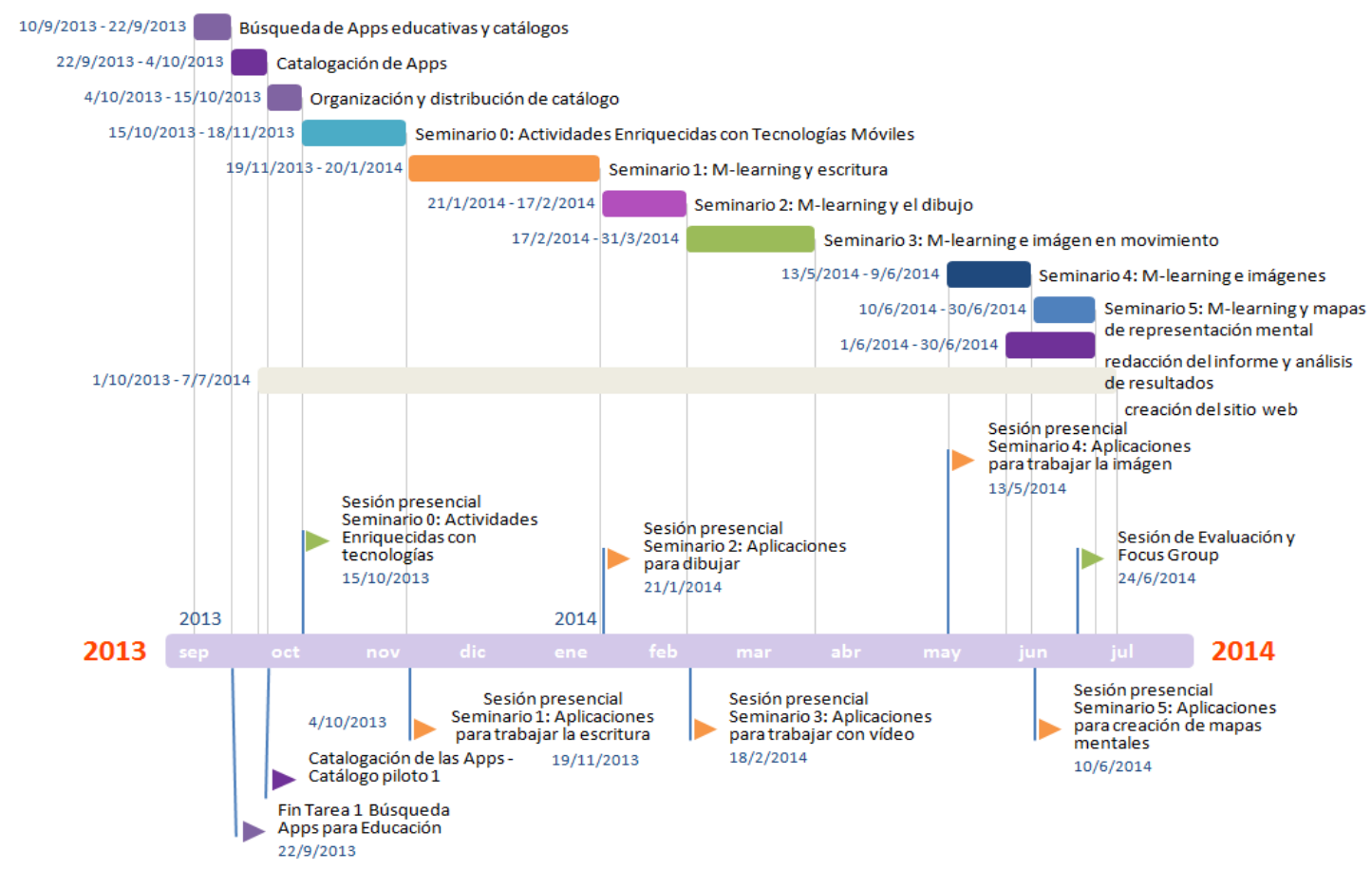

Figura 4. Cronología de trabajo

\section{RESULTADOS PRELIMINARES}

En este apartado mostramos los resultados preliminares del estudio en relación a la satisfacción de los participantes, los seminarios de formación, el diseño e implementación de las actividades, asesoramiento recibido y la valoración general. Estos resultados contribuyen a tratar de conocer el impacto del plan de formación.

El grupo de investigadores del GITE fue el encargado de realizar el seguimiento y evaluación de cada uno de los seminarios.

Los maestros cumplimentaron el cuestionario antes del inicio del siguiente seminario de formación. Por lo tanto, cada maestro lo cumplimentó tantas veces como sesiones hubo, un total de 5.

El análisis de datos fue realizado desde la propia herramienta "Encuestas" contemplándose estadísticos descriptivos.

El cuestionario fue cumplimentado en un total de 21 ocasiones, el $76 \%$ de las mismas fue llevado a cabo por docentes de sexo femenino.

Los maestros tuvieron la posibilidad de valorar una serie de actuaciones realizadas durante cada uno de los seminarios de formación. Para el equipo docente resultaron actuaciones adecuadas o muy adecuadas: lluvia de ideas, selección de 4 Apps por parte del formador, crear una actividad por cada aplicación, mostrar ejemplos con el uso de Apps (preparadas por el formador) y el asesoramiento del responsable del seminario. 
En la gráfica 1 se puede observar la valoración de la calidad de los seminarios en función a una serie de elementos. Fueron valorados positivamente como alta o muy alta los siguientes puntos: relevancia para su labor como docente $(A)$; posibilidad de aplicación práctica (B); clarificación de los objetivos (C); duración (D); método de trabajo (E); estructura (F); y complejidad de las tareas (G).

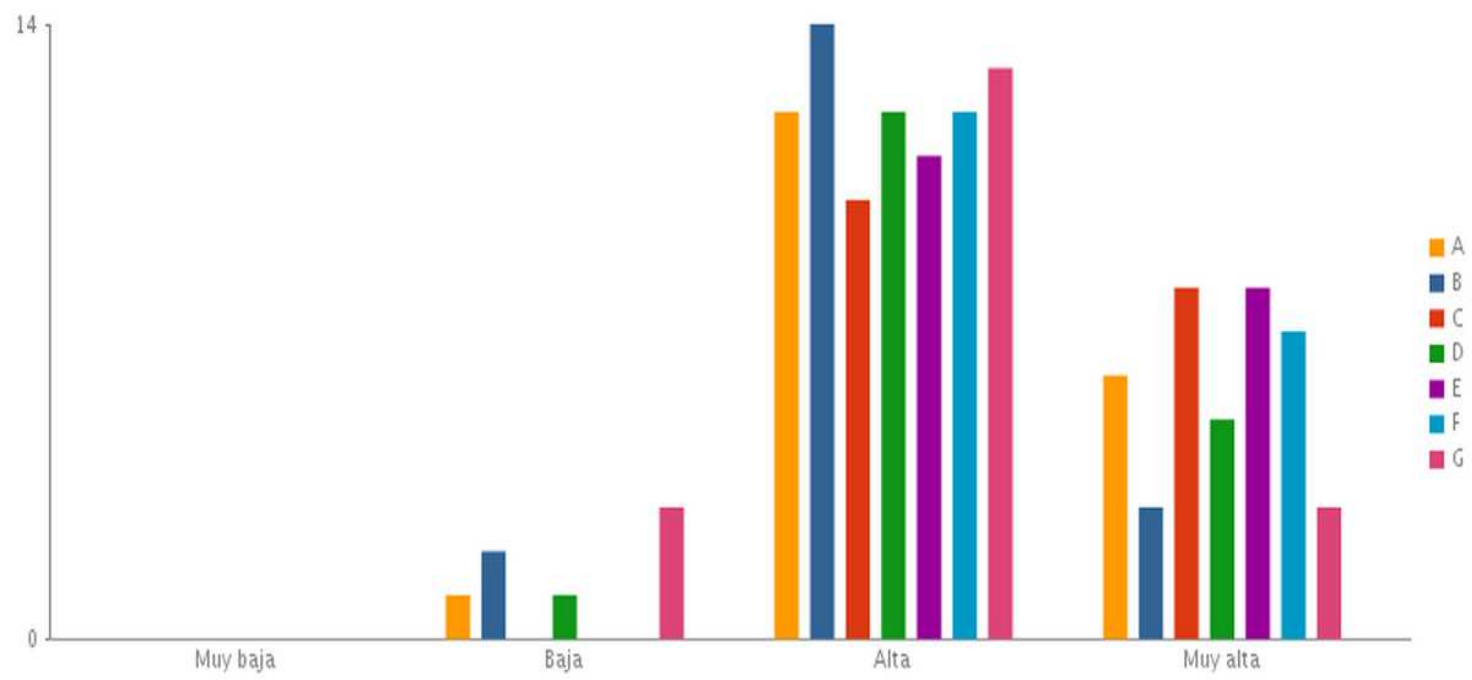

Gráfica 1. Valoración de la calidad de los seminarios de formación

En cuanto al grado de dificultad en el diseño de las actividades con el uso de Apps, encontramos resultados dispares y diversos. En la gráfica 2 queda reflejada dicha diversidad de respuestas. Los elementos consultados fueron: manejo técnico de la tablet $(A)$; manejo técnico del portátil $(B)$; manejor técnico de las Apps (C); tiempo disponible (D); conexión a Internet $(E)$; formación recibida $(F)$; y asesoramiento y apoyo recibido $(G)$.

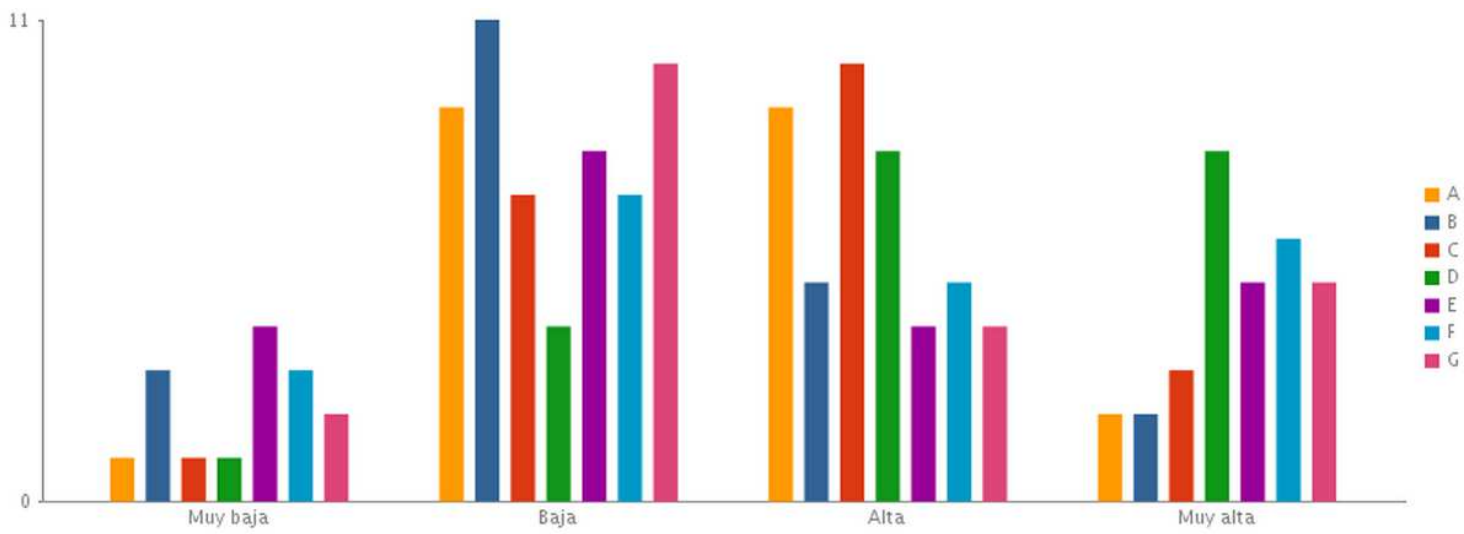

Gráfica 2. Grado de dificultad en el diseño de las actividades con el uso de Apps

Los docentes opinaron y valoraron el efecto que las TIC ha podido tener sobre el aprendizaje de sus estudiantes gracias a las actividades diseñadas durante los seminarios. De esta manera consideran que están más motivados e implicados en clase (19 respuestas), saben buscar información en distintas fuentes y recursos (16 
respuestas) y que han mejorado sus habilidades con las TIC (20 respuestas). Por el contrario, no consideran que hayan mejorado su rendimiento (16 respuestas) ni que trabajen más en equipo y de forma colaborativa (14 respuestas).

En la mayoría de las ocasiones los maestros consideraron que contar con el asesoramiento de los formadores en los distintos seminarios es necesario (46\%) o muy necesario (33\%).

Sin embargo, en 12 ocasiones de las 21 que se cumplimentó el cuestionario, los maestros afirmaron que no hicieron ninguna consulta durante todo el periodo de formación. Solamente en 9 ocasiones los maestros hicieron consultas pero pocas.

En líneas generales los maestros valoraron positivamente la experiencia de formación recibida (Gráfica 5), y más concretamente en relación con los siguientes elementos: seminario de formación presencial (finalidad, estructura, formador, contenidos) (A); proceso sugerido de diseño de actividades (B); asesoramiento ofrecido por el formador después del seminario (C); aplicabilidad de los aprendido (D).

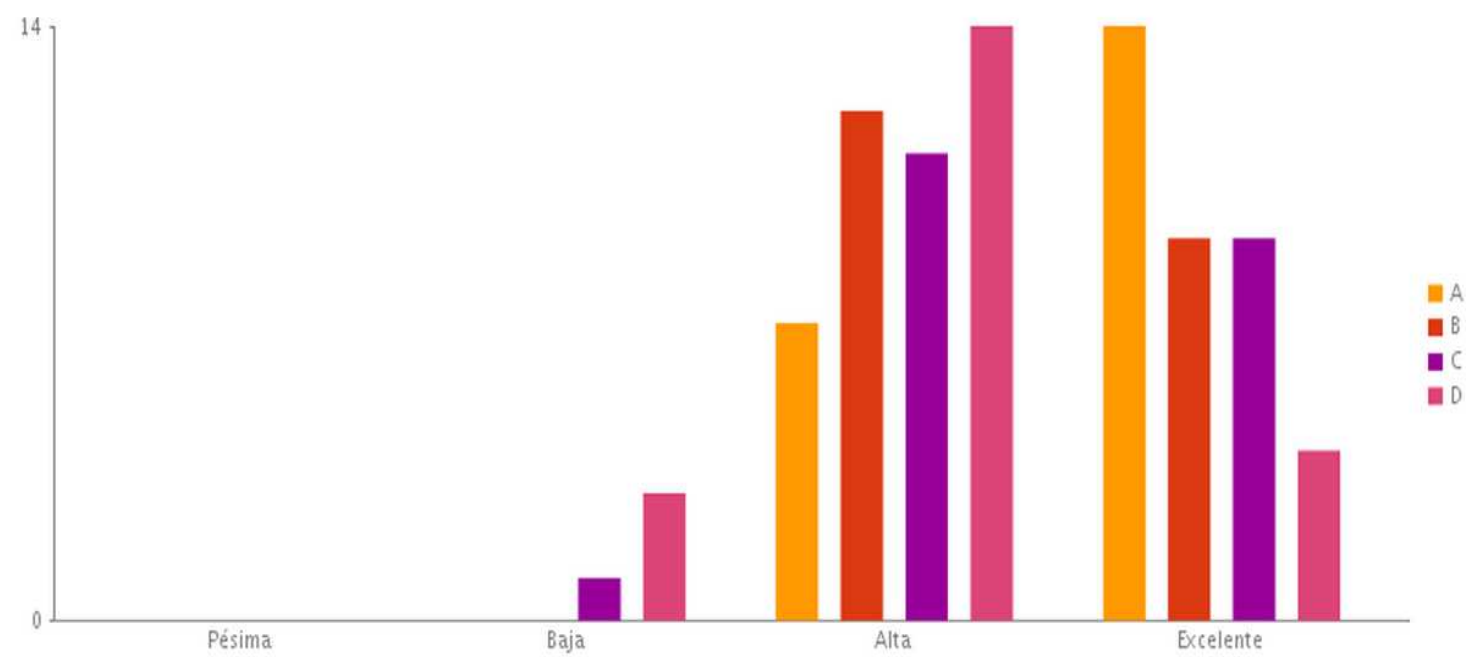

Gráfica 5. Valoración de la experiencia de formación

Para finalizar, y según las respuestas de los maestros, les gustaría recibir más formación sobre: aplicaciones para tablets (15); herramientas para el ordenador/portátil (10) y diseño de actividades con TIC (10).

\section{CONCLUSIONES}

Al amparo de este proyecto, se ha creado, depurado y publicado un metacatálogo de Apps. Incluye más de 380 aplicaciones probadas (119 de texto, 32 para dibujo, 145 para imagen fija, 5 para presentaciones, 21 mapa mentales, 57 vídeo y 2 de videoconferencia). Se ha convertido además en una herramienta online clasificada y buscable desde la red: http://goo.gl/22hUuA 
Como segundo hito principal, se ha diseñado, implementado y además evaluado un proceso de desarrollo profesional docente que ha tenido un grado muy alto de aceptación y satisfacción por parte de los maestros (en lo que se refiere especialmente a su utilidad).

Finalmente, se ha construido un catálogo de 47 actividades didácticas enriquecidas con tecnologías móviles, especialmente diseñadas para ser utilizadas en el contexto de las AAHH.

Además, se ha puesto a disposición de cualquier persona interesada: http://goo.gl//ElzJr

Estos resultados son la evidencia del logro de los objetivos previstos en el Proyecto EDUMOBSPITALARIOS.

Indicamos a continuación algunas conclusiones en relación a los resultados mostrados en el apartado anterior.

La calidad de los seminarios que formaron el plan de formación fue considerada muy positivamente por los docentes. Se considera relevante (los datos así lo dicen) el formato seguido en este plan de formación, tanto en su duración (un seminario por mes) como en su estructura (descrita en apartados anteriores).

La aplicación práctica del plan de desarrollo profesional docente fue valorada también muy positivamente, cumpliendo así una de las premisas del equipo de trabajo.

Los procesos de diseño, almacenamiento y distribución de las actividades han sido considerados como muy relevantes. Si bien es cierto que esto supone una carga adicional de trabajo, los maestros valoraron positivamente la posibilidad de colaborar con sus compañeros y ofrecer de manera abierta a cualquier usuario de Internet el trabajo realizado.

Según la opinión de los maestros, este plan de formación ha tenido un impacto directo en los alumnos hospitalizados. Consideran que se encuentran más motivados y que han mejorado sus habilidades con las TIC. Por el contrario afirman que el rendimiento académico del alumnado no ha mejorado como consecuencia directa del plan de desarrollo profesional.

Finalmente, en relación con el asesoramiento se ha podido observar en los datos que los docentes valoran positivamente el tipo de ayuda externa recibida por parte de los investigadores, siendo el tipo de asesoramiento más idóneo el que combina lo presencial y lo virtual. 


\section{DISCUSIÓN}

La formación de los docentes del hospital es en general uno de los pilares básicos sobre los que se viene realizando más desarrollos durante los últimos años, y en especial en el uso de TIC en AAHH. Las demandas de los propios maestros han propiciado el surgimiento de nuevas iniciativas basadas en planes de formación para la integración educativa de TIC. Experiencias que son llevadas a cabo en no pocas ocasiones entre equipos formados por maestros de $\mathrm{AAHH}$ e investigadores de diferentes grupos de investigación (González, 2015; Prendes, 2014; Violant, Cardone de Bove, Monsalve y Márques, 2012).

Desde el año 2010 el grupo de maestros del EAEHD y el grupo de investigadores del GITE han colaborado para trabajar en una de las principales claves en la integración de las TIC en el aula: la configuración de equipos externos de apoyo a los centros educativos para facilitar la tarea de coordinación de proyectos y la resolución de problemas reales (Area, 2002).

En este sentido hemos podido volver a constatar que la formación y el asesoramiento es una de las dimensiones claves en los procesos de integración curricular de TIC en AAHH (Serrano y Prendes, 2015). Por lo tanto se vuelve a tener evidencias sobre la recomendación de que existan figuras externas responsables de la formación que ofrezcan un asesoramiento continuo para apoyar el proceso de integración de TIC.

El m-learning es actualmente una de las tendencias de investigación en tecnología educativa, aunque en realidad lo viene siendo desde principios de siglo XXI (Hsu, 2012). Se ha investigado desde diferentes perspectivas: su relación con las inteligencias múltiples (Santiago, Amo y Díez, 2014), la producción de recursos (Sandoval, García y Ramírez, 2012), detección de emociones en el aprendiz (Ally \& Prieto-Blázquez, 2014), su relación con las habilidades cognitivas superiores (Ramos, Herrera, Ramírez, 2010), etc.

Según la exploración realizada por Baran (2014) la mayoría de las investigaciones publicadas hasta la fecha se centraron principalmente en el alumnado. Sin embargo, y coincidiendo con nuestra experiencia, existe una tendencia a investigar sobre el potencial del m-learning dentro de la formación del profesorado. Según el autor, se precisa el desarrollo de líneas de investigación para el diseño de modelos teóricos que sugieran cómo integrar el m-learning en la formación del profesorado. Además del modelo presentado en este artículo, existen otras recientes experiencias (por ejemplo la presentada en Madrid, Mayorga y Núñez, 2013) que auguran el aumento del conocimiento en esta dirección.

En Serrano y Prendes (2015) ya se mostraron una serie de líneas futuras de investigación en relación al uso de TIC en AAHH. Sin embargo se indica de manera adicional una propuesta más derivada del estudio presentado en este artículo. Se trata de la posible realización de un análisis exhaustivo de las actividades diseñadas en el marco del proyecto y que se encuentran recogidas en el catálogo presentado anteriormente. Sería de interés analizar las características de estas actividades 
estableciendo patrones de calidad.

\section{AGRADECIMIENTOS}

Colaborar con otros profesionales con diferentes necesidades, inquietudes, concepciones y realidades, no es tarea sencilla. Sin embargo cuando existe una actitud de colaboración tan valiosa como la del equipo de maestros del EAEHD esta tarea se convierte es una excelente oportunidad de aprendizaje sin límites. Agradecemos a este equipo la confianza depositada en los investigadores del GITE para tratar de ayudar en la meta de las $\mathrm{AAHH}$ : mejorar la calidad de vida del alumnado hospitalizado mediante el aprendizaje.

\section{REFERENCIAS}

ADELL, J. Y CASTAÑEDA, L. (2013). El ecosistema pedagógico de los PLEs. En L.

CASTAÑEDA Y J. ADELL (Eds.), Entornos Personales de Aprendizaje: Claves para el ecosistema educativo en red (pp. 29-51). Alcoy: Marfil.

AREA, M. (2002). La integración escolar de las nuevas tecnologías. Entre el deseo y la realidad. Organización y gestión educativa, 6, 14-18.

BARAN, E. (2014). A Review of Research on Mobile Learning in Teacher Education. Educational Technology \& Society, 17 (4).

CAMPBELL, L. Y ST LEGER, P. (2006). On the Right Track: An evaluation of the Back on Track pilot program on behalf of the Royal Children's Hospital Education Institute. Melbourne. Centre for Program Evaluation, University of Melbourne: Children's Hospital Education Institute.

CASTAÑEDA, L. Y ADELL, J. (2011). El desarrollo profesional de los docentes en entornos personales de aprendizaje (PLE). En Roig Vila, R. y Laneve, C. (Eds.) La práctica educativa en la Sociedad de la Información: Innovación a través de la investigación / La pratica educativa nella Società dell'informazione: L'innovazione attraverso la ricerca. Alcoy: Marfil. (pp. 83-95) Disponible en http://digitum.um.es/xmlui/bitstream/10201/24647/1/CastanedaAdell2011prep rint.pdf

CASTAÑEDA, L. Y SÁNCHEZ, M.M. (2012). Realidades TIC en las aulas hospitalarias: experiencias de uso y algunas buenas prácticas. En M.P. Prendes, J.L. Serrano (Coords.), Las TIC en las aulas hospitalarias (pp.53-70). Alicante: Marfil.

COOK, R. (2005). Evaluation of the WellCONNECTED pilot project: The use of Information and Communication Technologies in secondary schools in Victoria to 
maintain and strengthen the connection to school for senior students with a chronic health condition. Melbourne: Royal.

FULLAN, M. (2002). Los nuevos significados del cambio en la Educación. Barcelona: Octaedro.

GONZÁLEZ, C.S. (2015). Las TIC como apoyo al aula hospitalaria. En C.S. González y V. Violant, Uso de las TIC para la atención educativa hospitalaria y domiciliaria. Madrid: McGraw-Hill Interamericana de España.

GONZÁLEZ, C.S., GONZÁLEZ, S., LILIANA, M., HERNÁNDEZ, G., TOLEDO, P. Y AREA, M. (2011). Diseño de espacios virtuales para la comunicación y la atención educativa hospitalaria. En C.S. González, Investigación e innovación en Tecnologías Aplicadas a la Educación. Actividades y experiencias en I+D+i, (pp. 170-187). España: Bubok Publishing.

HSU, Y.-C., HO, H.N.J., TSAI, C.-C., HWANG, G.-J., CHU, H.-C., WANG, C.-Y. \& CHEN, N.-S. (2012). Research Trends in Technology-based Learning from 2000 to 2009: A content Analysis of Publications in Selected Journals. Educational Technology \& Society, 15 (2), 354-370.

JONES, A. Y MCDOUGALL, A. (2010). Helping students with a chronic illness connect to their teachers and school. En D. Gronn y G. Romeo (Eds.), Conference Proceedings of the Australian Computers in Education. Melbourne: Australian Council for Computers in Education. Extraído el 25 de octubre de 2013 de http://acec2010.acce.edu.au/proposal/298/helping-students-chronicillnessconnect-their-teachers-and-school

MADRID, D., MAYORGA, M.J. \& NÚÑEZ, F. (2013). Aplicación del m-learning en el aula de primaria: experiencia práctica y propuesta de formación para docentes. EDUTEC, Revista Electrónica de Tecnología Educativa, 45. Recuperado el 28/07/2015 de http://edutec.rediris.es/Revelec2/Revelec45/aplicacion_mlearning_primaria_exp eriencia_formacion_docentes.html

NISSELLE A., HANNS S., GREEN J. Y JONES T. (2012). Accessing fl exible learning opportunities: patients' use of laptops in a paediatric hospital. Technology, Pedagogy \& Education, 21(1) 3-20.

PRENDES, M.P. (Dir) (2014). ALTER. Alternativas telemáticas en Aulas Hospitalarias, una experiencia educativa. Informe del Proyeto EDU2008-01921 de la Fundación Séneca, Región de Murcia. Recuperado el 20/06/2015 de http://www.um.es/aulashospitalarias/informe.php

PRENDES, M.P., CASTAÑEDA, L. Y SERRANO, J.L. (2014). Entre la colaboración y la formación: un modelo de incorporación de tecnologías en las Aula Hospitalarias de la Región de Murcia. Comunicación y pedagogía: Nuevas tecnologías y recursos didácticos, 279-280, 92-98. 
RAMOS, A.I., HERRERA, J.A. Y RAMÍREZ, M.S. (2010). Desarrollo de habilidades cognitivas con aprendizaje móvil: un estudio de casos. Comunicar, 34 (17), 201209.

SANDOVAL, E.A., GARCÍA, R. \& RAMÍREZ, M.S. (2012). Competencias tecnológicas y de contenido necesarias para capacitar en la producción de recursos de aprendizaje móvil. EDUTEC, Revista Electrónica de Tecnología Educativa, 39. Recuperado el 22/07/2015 http://edutec.rediris.es/Revelec2/Revelec39/competencias_tecnologicas_conteni do_capacitacitar_produccion_recursos_aprendizaje_movil.html

SANTIAGO, R., AMO, D. \& DÍEZ, A. (2014) ¿Pueden las aplicaciones educativas de los dispositivos móviles ayudar al desarrollo de las inteligencias múltiples? EDUTEC, Revista Electrónica de Tecnología Educativa, 47. Recuperado el 29/07/2015 de http://edutec.rediris.es/Revelec2/Revelec47/n47_Santiago-Amo-Diez.html

SERRANO, J. L. Y PRENDES, M. P. (2014). TIC para la mejora educativa en aulas hospitalarias. Píxel-Bit. Revista de Medios y Educación,45, 23-36.

SERRANO, J.L. (2013). Herramientas telemáticas en aulas hospitalarias: una experiencia educativa en la Región de Murcia. Tesis Doctoral. Universidad de las Islas Baleares, España. Recuperado el 09/09/2013 de http://digitum.um.es/xmlui/bitstream/10201/35643/1/tjss1de1.pdf

SERRANO, J.L. Y LÓPEZ, M.P. (2014). Actividades enriquecidas con el uso de vídeos en aulas hospitalarias. Congreso Internacional EDUTEC 2014. Córdoba, España. Recuperado el 01/08/2015 de http://hdl.handle.net/10201/41466

SERRANO, J.L. Y PRENDES, M.P. (2015). Integración de TIC en aulas hospitalarias como recursos para la mejora de los procesos educativos. Estudios Sobre Educación, $28,187-210$.

VIOLANT, V., CARDONE DE BOVE, P., MONSAlVE, C. Y MÁRQUES, L. (2012). Multidimensionalidad de la pedagogía hospitalaria desde la transdisciplinariedad: análisis de necesidades de los itinerarios formativos. En IV Forum Internacional innovación y creatividad. Adversidad y escuelas creativas (pp. 1-9). Barcelona, España. Recuperado el 01/06/2015 de http://hdl.handle.net/2445/34520

WILLIAMS, R., MACKNESS, J. Y GUMTAU, S. (2012). Footprints of emergence. The International of Research in Open and Distance Learning, 13(4). Disponible en http://www.irrodl.org/index.php/irrodl/article/view/1267/230

Para citar este articulo:

Serrano J. L. \& Castañeda L. (2016). Proyecto edumobspitalarios: desarrollo profesional docente e innovación con m-learning en aulas hospitalarias. EDUTEC, Revista Electrónica de Tecnología Educativa, 55. Recuperado el dd/mm/aa de http://www.edutec.es/revista 